\title{
Commentary: It's time to check our bias: Ensuring health equity for patients with lung cancer
}

\author{
Pamela Samson, MD, MPHS
}

From the Department of Radiation Oncology, Washington University in St Louis, St Louis, Mo.

Disclosures: Author has nothing to disclose with regard to commercial support.

Received for publication Dec 17, 2018; accepted for publication Dec 17, 2018; available ahead of print Feb 6, 2019.

Address for reprints: Pamela Samson, MD, MPHS, Department of Radiation Oncology, Washington University in St Louis, 4511 Forest Park Ave, St Louis, MO 63108 (E-mail: psamson@wustl.edu).

J Thorac Cardiovasc Surg 2019;157:1680-1

$0022-5223 / \$ 36.00$

Copyright (c) 2018 by The American Association for Thoracic Surgery

https://doi.org/10.1016/j.jtcvs.2018.12.058

Wolf and colleagues ${ }^{1}$ examine the Surveillance, Epidemiology, and End Results Medicare registry to examine treatment disparities for stage I non-small cell lung cancer (NSCLC) between white and black patients. This study broadens our knowledge of inequality in lung cancer treatment among Medicare-insured patients by finding that black patients were less likely to receive any treatment for early-stage NSCLC or receive surgery, and they were more likely to refuse surgery than white patients. Although 5-year overall survival was lower for black patients, adjusting survival for the treatment type delivered showed no differences by race. It is challenging to identify factors driving these associations in large databases, but the authors did investigate possible interactions and determined that these findings were not significantly confounded by comorbidities. Whereas a limitation of the data is the time period (1992-2009), the work is valuable in providing a baseline

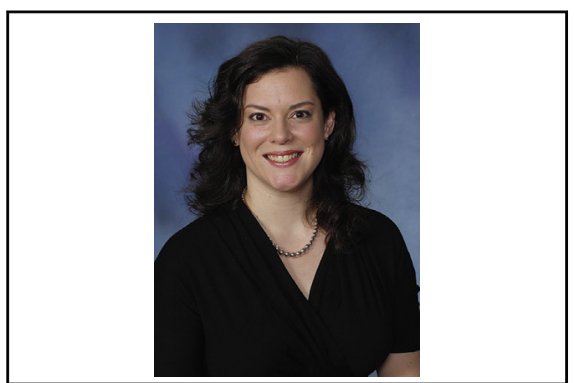

Illustration of how equity promotes similar outcomes (ie, seeing the game) versus equality.

\section{Central Message}

Among Medicare patients, lower rates of treatment uptake for stage I non-small cell lung cancer persist for black patients. Identifying and acting on barriers to care will support health equity.

See Article page 1670.

of differential treatments that can be used in the future to gauge improvement or alert us to deepening divides. It is important to note that of 21,000 patients in this analysis, only $6.6 \%$ were black. Current Surveillance, Epidemiology, and End Results statistics show that black men

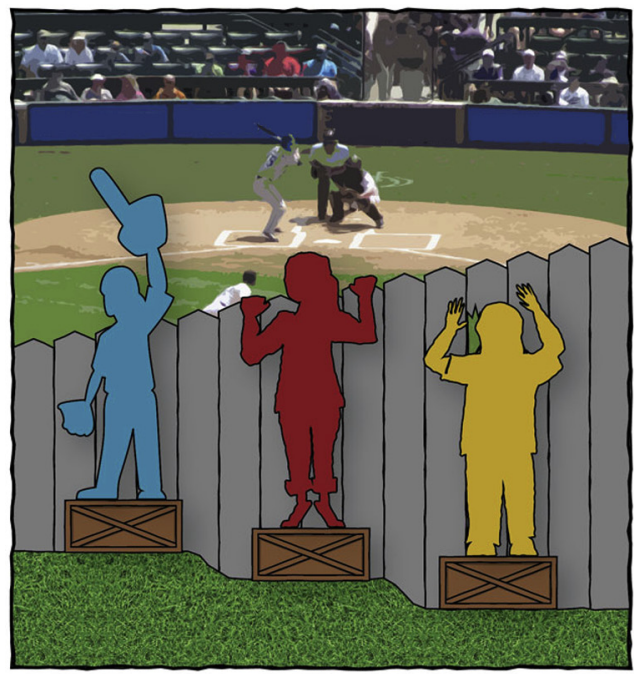

EQUALITY

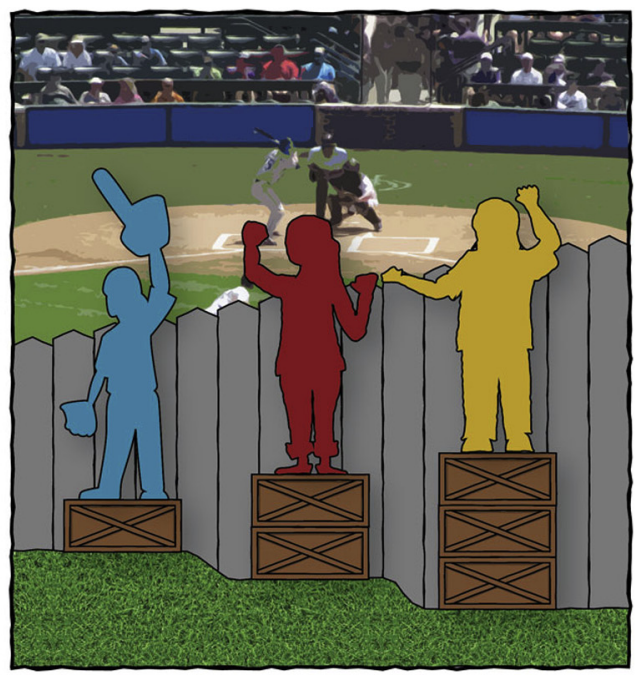

EQUITY

FIGURE 1. Illustration of how equity promotes similar outcomes (ie, seeing the game) versus equality. Reprinted with permission from Dr Paul Kuttner. ${ }^{3}$ 
actually have the highest rate of new lung cancer cases (81.2 per 100,000 persons vs 63.9 for white men); therefore, this discrepancy in the stage I database is concerning for presentation at advanced stages. ${ }^{2}$

So what can we do with this information and what practical framework do we use to improve these disparities? An important consideration is acknowledging the difference between health equality and equity. A popular graphic illustrates the differences in these terms (Figure 1). ${ }^{3}$ In health equality, physicians and staff identify conscious and unconscious racial biases and work to understand how these may influence treatment allocation and approaches. The most frequently cited test has been the Implicit Association Test, which has documented racial bias in physicians at similar rates to the general population. ${ }^{4}$

For health equity, we need to identify strategies to get all patients to the same finish line of definitive therapy for early stage NSCLC. This involves understanding what barriers may exist for patients, especially for populations that have historically been marginalized or discriminated against by the health care system, and finding ways to bridge that gap. The potential barriers are numerous: social, economic, cultural, logistical, and historical to name a few. A single person sitting before us in our office may have none, 1 , or all of these barriers that could influence his or her ability to proceed with treatment, but if we do not ask about his or her concerns and listen, we will miss opportunities to ensure equity. For example, if major concerns for a patient are driven by socioeconomic factors, the introduction of patient navigators, social work, and/or financial counseling resources may make a substantial difference in our ability to provide care. If the driving concern is historical (eg, previous interactions with the health care system), we have no greater opportunity to address that barrier than in that very moment.

\section{References}

1. Wolf A, Alpert N, Tran BV, Liu B, Flores R, Taioli E. Persistence of racial disparities in early-stage lung cancer treatment. J Thorac Cardiovasc Surg. 2019;157:1670-9.e4.

2. Surveillance, Epidemiology, and End Results Program. Cancer stat facts: lung and bronchus cancer. Available at: https://seer.cancer.gov/statfacts/html/lungb.html. Accessed December 17, 2018.

3. Kuttner P. The problem with that equity versus equality graphic you're using Available at: http://culturalorganizing.org/the-problem-with-that-equity-vsequality-graphic/. Accessed December 17, 2018.

4. Hall WJ, Chapman MV, Lee KM, Merino YM, Thomas TW, Payne BK, et al Implicit racial/ethnic bias among health care professionals and its influence on health care outcomes: a systematic review. Am J Public Health. 2015;105: e60-76. 